\title{
CRITERION FOR THE RESOLVENT SET OF NONSYMMETRIC TRIDIAGONAL OPERATORS
}

\author{
A. I. APTEKAREV, V. KALIAGUINE, AND W. VAN ASSCHE
}

(Communicated by Theodore W. Gamelin)

\begin{abstract}
We study nonsymmetric tridiagonal operators acting in the Hilbert space $\ell^{2}$ and describe the spectrum and the resolvent set of such operators in terms of a continued fraction related to the resolvent. In this way we establish a connection between Padé approximants and spectral properties of nonsymmetric tridiagonal operators.
\end{abstract}

\section{INTRODUCTION}

In this paper we consider operators in the Hilbert space $\ell^{2}$, with the following representation in an orthonormal basis of this space:

$$
A=\left(\begin{array}{ccccc}
\beta_{0} & \gamma_{0} & 0 & 0 & \ldots \\
\alpha_{1} & \beta_{1} & \gamma_{1} & 0 & \ldots \\
0 & \alpha_{2} & \beta_{2} & \gamma_{2} & \ldots \\
\vdots & \vdots & \ddots & \ddots & \ddots
\end{array}\right)
$$

where $\alpha_{k}, \beta_{k}, \gamma_{k}$ are complex numbers, $\alpha_{k} \neq 0, \gamma_{k} \neq 0, k \in \mathbb{N}$. The operator $A$ is defined for any finite vector $x=x_{0} g_{0}+x_{1} g_{1}+\cdots+x_{n} g_{n}$ in the orthonormal basis $\left\{g_{n}\right\}_{0}^{\infty}$, and its domain of definition $D(A)$ is dense in $\ell^{2}$.

The symmetric case, where $\alpha_{k}=\overline{\gamma_{k-1}}$ and $\beta_{k}$ is real, can under an appropriate chosen basis be reduced to an infinite Jacobi matrix enabling a deeper examination of this spectral theory. The main tools of investigation in this case are the classical moment problem (including the theory of selfadjoint extensions of unbounded symmetric operators), the theory of general orthogonal polynomials and the spectral theorem for selfadjoint operators ([1], [2], [9], [11]). This connection between the spectral theory and analysis is fruitful for various points of view. For example, the scattering problem for a Jacobi matrix can be treated on the basis of strong (or Szegö type) asymptotic results for orthogonal polynomials ([4], [6], [10]). On the other hand, the perturbation theory gives new results for orthogonal polynomials ([12]).

Received by the editors April 7, 1993 and, in revised form, November 23, 1993.

1991 Mathematics Subject Classification. Primary 47A10, 41A21.

Key words and phrases. Tridiagonal operators, resolvents, Padé approximation.

The first author is partly supported by the Russian Basic Research Foundation (grant 93-01278); the third author is a Senior Research Associate of the Belgian National Fund for Scientific Research. 
In the general, nonsymmetric, case we cannot use the spectral theorem and orthogonal polynomials, but in return we will obtain results of use for Padé approximants and the theory of continued fractions. We denote as usual by $\sigma(A)$ the spectrum of the operator $A, \Omega(A)=\mathbb{C} \backslash \sigma(A)$ is the resolvent set and the resolvent is $R(\lambda)=(\lambda I-A)^{-1}$ for $\lambda \in \Omega$. By $\left\{g_{n}\right\}_{0}^{\infty}$ we denote the orthonormal basis in $\ell^{2}$. The function

$$
\phi(\lambda)=\left(R(\lambda) g_{0}, g_{0}\right)
$$

is analytic on the resolvent set $\Omega(A)$. If the operator $A$ is bounded (this is the case when $\alpha_{k}, \beta_{k}, \gamma_{k}$ are bounded), then the function $\phi(\lambda)$ is analytic for $|\lambda|>\|A\|$. For convenience we have changed the basis of representation of the operator $A$. The new basis $\left\{e_{n}\right\}_{0}^{\infty}$ is defined by $e_{n}=g_{n} / d_{n}$, where $d_{n}=$ $\gamma_{0} \gamma_{1} \ldots \gamma_{n-1}, d_{0}=1$. In this basis the operator (1) has the following form:

$$
A=\left(\begin{array}{ccccc}
\beta_{0} & 1 & 0 & 0 & \ldots \\
\alpha_{1} \gamma_{0} & \beta_{1} & 1 & 0 & \ldots \\
0 & \alpha_{2} \gamma_{1} & \beta_{2} & 1 & \ldots \\
\vdots & \vdots & \ddots & \ddots & \ddots
\end{array}\right)
$$

If we set $a_{n}=\alpha_{n} \gamma_{n-1}, b_{n}=\beta_{n}$, then we have in the basis $\left\{e_{n}\right\}$ :

$$
A=\left(\begin{array}{ccccc}
b_{0} & 1 & 0 & 0 & \ldots \\
a_{1} & b_{1} & 1 & 0 & \ldots \\
0 & a_{2} & b_{2} & 1 & \ldots \\
\vdots & \vdots & \ddots & \ddots & \ddots
\end{array}\right) .
$$

The Chebyshev algorithm (Algorithm 7.2.1 in [8, page 248]) applied to $\phi(\lambda)$ at infinity gives us the following continued fraction ( $\mathrm{J}$-fraction):

$$
\frac{1 \mid}{\mid \lambda-b_{0}}-\frac{a_{1} \mid}{\mid \lambda-b_{1}}-\frac{a_{2} \mid}{\mid \lambda-b_{2}}-\cdots \text {. }
$$

The numerators $P_{n}$ and denominators $Q_{n}$ of the $n$th convergents for this fraction satisfy the three-term recurrence relation:

$$
a_{n} y_{n-1}+b_{n} y_{n}+y_{n+1}=\lambda y_{n}, \quad n=0,1,2, \ldots,
$$

with the initial conditions

$$
\begin{cases}Q_{-1}=0, & Q_{0}=1, \\ P_{-1}=1, & P_{0}=0 .\end{cases}
$$

The main problem considered in this paper is to describe the spectrum and the resolvent set of operators of type (1) in terms of the continued fraction (5) and the monic polynomials $P_{n}, Q_{n}$ and in this way to establish a connection between Padé approximants and spectral properties of nonsymmetric tridiagonal operators. In our main result, Theorem 1 , we state the criterion for the resolvent set $\Omega(A)$ in terms of the growth of the polynomials $Q_{n}(z)$. The proof of the necessary condition is essentially based on the results of Kershaw and Demko, Moss, and Smith about decay rates of inverses of banded matrices ([5]). This theorem gives us analytic properties of the polynomials $Q_{n}(\lambda)$ and the related Padé approximants $\pi_{n}:=P_{n} / Q_{n}$ on the set $\Omega(A)$. We prove, among other things, the following 
Theorem 2. If $A$ is bounded and

$$
0<\left|\alpha_{k}\right|,\left|\gamma_{k}\right| \leq C_{1}<\infty
$$

for some $C_{1}$, then for any $\lambda \in \Omega(A)$ there is a subsequence of Padé approximants $\pi_{n}$ which converges to $\phi(\lambda)$ with a geometric rate.

In section 2 we formulate and prove Theorem 1 and related results. Section 3 will be devoted to an application of these results to the behavior of Padé approximants.

\section{ANALYSIS OF THE SPECTRUM AND THE RESOLVENT SET OF THE OPERATOR}

Suppose $A$ has a representation (4) in an orthogonal basis $\left\{e_{n}\right\}$, where $\left\|e_{n}\right\|=h_{n}$. We assume that the operator $A$ is bounded. In this case $A$ is defined on the whole space $\ell^{2}$ and $\lambda \in \Omega(A)$ if and only if the following conditions are satisfied:

(1) $\operatorname{Ker}(\lambda I-A)=\{0\}$,

(2) $\forall n, \exists z_{n}:(\lambda I-A) z_{n}=e_{n}$,

(3) $\left\|(\lambda I-A)^{-1} x\right\| \leq C\|x\|$, for all finite vectors $x=x_{0} e_{0}+x_{1} e_{1}+\cdots+x_{n} e_{n}$ and some constant $C$.

Proposition 1. $\lambda_{0}$ is an eigenvalue of $A$ if and only if

$$
\sum_{n=0}^{\infty}\left|Q_{n}\left(\lambda_{0}\right)\right|^{2} h_{n}^{2}<+\infty .
$$

Proof. We can formally write $A x=\lambda_{0} x$ and obtain for $x=x_{0} e_{0}+x_{1} e_{1}+\cdots$ the following system:

$$
\left\{\begin{array}{ccc}
b_{0} x_{0}+x_{1} & =\lambda_{0} x_{0} \\
a_{1} x_{0}+b_{1} x_{1}+x_{2} & =\lambda_{0} x_{1} \\
a_{2} x_{1}+b_{2} x_{2}+x_{3} & =\lambda_{0} x_{2} \\
\vdots & \vdots & \vdots
\end{array}\right.
$$

If $x_{0}=0$, then $x_{n}=0, \forall n \in \mathbb{N}$. If $x_{0} \neq 0$, then $x_{n}=Q_{n}\left(\lambda_{0}\right) x_{0}$ and in this case $x \in \ell^{2}$ iff $\sum_{0}^{\infty}\left|Q_{n}\left(\lambda_{0}\right)\right|^{2} h_{n}^{2}<\infty$.

Proposition 2. For given $\lambda$, the equation

$$
(\lambda I-A) z=e_{n}
$$

has a solution in $\ell^{2}$ for all $n \geq 0$ if and only if there exists a complex number $\gamma$ such that

$$
\sum_{n=0}^{\infty}\left|Q_{n}(\lambda) \gamma-P_{n}(\lambda)\right|^{2} h_{n}^{2}<+\infty .
$$

Proof. First we find the formal solution $z^{(0)}$ of the equation

$$
(\lambda I-A) z=e_{0} \text {. }
$$

Let $z^{(0)}=\left(z_{0,0}, z_{1,0}, z_{2,0}, \ldots\right)$ in the basis $\left\{e_{n}\right\} ;$ then

$$
\left\{\begin{array}{cccc}
1+b_{0} z_{0,0}+z_{1,0} & =\lambda z_{0,0} \\
a_{1} z_{0,0}+b_{1} z_{1,0}+z_{2,0} & =\lambda z_{1,0} \\
a_{2} z_{1,0}+b_{2} z_{2,0}+z_{3,0} & =\lambda z_{2,0} \\
\vdots & \vdots & \vdots
\end{array}\right.
$$


So $z_{n, 0}$ satisfies the same recurrence relations as $Q_{n}(\lambda)$ and $P_{n}(\lambda)$ for $n \geq 1$, only the first relation is different. This implies that $z_{n, 0}=Q_{n}(\lambda) \gamma-P_{n}(\lambda)$ for $n>1$ and for any $\gamma$ the first relation is also satisfied: $b_{0} \gamma+\gamma\left(\lambda-b_{0}\right)-1+1=\lambda \gamma$. Thus the sequence $Q_{n}(\lambda) \gamma-P_{n}(\lambda)$ is a solution of (9) for any $\gamma$. We need a solution in $\ell^{2}$. So $e_{0}$ is in the image of $(\lambda I-A)$ iff $\sum_{0}^{\infty}\left|Q_{n}(\lambda) \gamma-P_{n}(\lambda)\right|^{2} h_{n}^{2}<$ $+\infty$ for some $\gamma$. Note that if $\lambda$ is not an eigenvalue of $A$, then the convergence of this series is possible only for one $\gamma$. For the solution of the equation $(\lambda I-A) z=e_{1}$ we note that $(\lambda I-A) e_{0}=\lambda e_{0}-A e_{0}=\left(\lambda-b_{0}\right) e_{0}-a_{1} e_{1}$ and then $-a_{1} e_{1}=(\lambda I-A)\left(e_{0}-\left(\lambda-b_{0}\right) z^{(0)}\right)$. So $z^{(1)}=-\left(e_{0}-\left(\lambda-b_{0}\right) z^{(0)}\right) / a_{1}$ is a solution of $(\lambda I-A) z=e_{1}$. This is an element of $\ell^{2}$ if $z^{(0)} \in \ell^{2}$. In the same way we find $z^{(2)}=-\left(e_{1}-\left(\lambda-b_{1}\right) z^{(1)}+z^{(0)}\right) / a_{2}$ for the solution of $(\lambda I-A) z=e_{2}$ and so on.

Theorem 1. Suppose $A$ is bounded, $\lambda$ is not an eigenvalue of $A$, and (8) is satisfied for some $\gamma$. Then $\lambda \in \Omega(A)$ if and only if there are constants $C>0$ and $0<q<1$ such that

$$
\begin{array}{ll}
\left|\frac{Q_{n}(\lambda) r_{m}(\lambda)}{a_{1} a_{2} \ldots a_{n}} \frac{h_{m}}{h_{n}}\right|, \leq C q^{m-n}, & n \leq m, \\
\left|\frac{Q_{m}(\lambda) r_{n}(\lambda)}{a_{1} a_{2} \ldots a_{n}} \frac{h_{m}}{h_{n}}\right| \leq C q^{n-m}, & n \geq m,
\end{array}
$$

where $r_{m}(\lambda):=Q_{m}(\lambda) \gamma-P_{m}(\lambda)$.

Proof. From Propositions 1 and 2 it follows that the inverse operator $B=$ $(\lambda I-A)^{-1}$ can be defined on the basis vectors by $B e_{n}=z^{(n)}$. The matrix of $B$ in the basis $\left\{e_{n}\right\}$ is of the form:

$$
\left(\begin{array}{ccccc}
r_{0} & \frac{r_{1}}{a_{1}} & \frac{r_{2}}{a_{1} a_{2}} & \frac{r_{3}}{a_{1} a_{2} a_{3}} & \ldots \\
r_{1} & Q_{1} \frac{r_{1}}{a_{1}} & Q_{1} \frac{r_{2}}{a_{1} a_{2}} & Q_{1} \frac{r_{3}}{a_{1} a_{2} a_{3}} & \ldots \\
r_{2} & r_{2} \frac{Q_{1}}{a_{1}} & Q_{2} \frac{r_{2}}{a_{1} a_{2}} & Q_{2} \frac{r_{3}}{a_{1} a_{2} a_{3}} & \ldots \\
r_{3} & r_{3} \frac{Q_{1}}{a_{1}} & r_{3} \frac{Q_{2}}{a_{1} a_{2}} & Q_{3} \frac{r_{3}}{a_{1} a_{2} a_{3}} & \ldots \\
\vdots & \vdots & \vdots & \vdots & \ddots
\end{array}\right) .
$$

Indeed, for the first column there is no problem, since it contains $z^{(0)}$. The $n$th column contains $z^{(n)}$. For $z^{(1)}=-\left(e_{0}-\left(\lambda-b_{0}\right) z^{(0)}\right) / a_{1}$ we have

$$
\begin{aligned}
& z_{0,1}=-\frac{1-\left(\lambda-b_{0}\right) r_{0}}{a_{1}}=\frac{r_{1}}{a_{1}} \\
& z_{1,1}=-\frac{-\left(\lambda-b_{0}\right) z_{1,0}}{a_{1}}=Q_{1} \frac{r_{1}}{a_{1}} \\
& z_{2,1}=-\frac{-\left(\lambda-b_{0}\right) z_{2,0}}{a_{1}}=r_{2} \frac{Q_{1}}{a_{1}}
\end{aligned}
$$

This is the second column of $B$. Suppose we have calculated $z^{(k)}$ as indicated and we have to find $z^{(k+1)}$. First note that

$$
z^{(k+1)}=\frac{e_{k}-\left(\lambda-b_{k}\right) z^{(k)}+z^{(k-1)}}{a_{k+1}} .
$$


Then

$$
\begin{gathered}
z_{0, k+1}=-\frac{1}{a_{k+1}}\left(-\left(\lambda-b_{k}\right) \frac{r_{k}}{a_{1} a_{2} \cdots a_{k}}+\frac{r_{k-1}}{a_{1} a_{2} \cdots a_{k-1}}\right)=\frac{r_{k+1}}{a_{1} a_{2} \cdots a_{k+1}} \\
z_{1, k+1}=-\frac{1}{a_{k+1}}\left(-\left(\lambda-b_{k}\right) \frac{Q_{1} r_{k}}{a_{1} a_{2} \cdots a_{k}}+\frac{Q_{1} r_{k-1}}{a_{1} a_{2} \cdots a_{k-1}}\right)=Q_{1} \frac{r_{k+1}}{a_{1} a_{2} \cdots a_{k+1}} \\
\quad \vdots \quad \vdots \\
z_{k, k+1}=-\frac{1}{a_{k+1}}\left(1-\left(\lambda-b_{k}\right) \frac{Q_{k} r_{k}}{a_{1} a_{2} \cdots a_{k}}+\frac{Q_{k-1} r_{k}}{a_{1} a_{2} \cdots a_{k-1}}\right)=Q_{k} \frac{r_{k+1}}{a_{1} a_{2} \cdots a_{k+1}}
\end{gathered}
$$

We used here the relations $Q_{k-1} r_{k}-Q_{k} r_{k-1}=-a_{1} a_{2} \cdots a_{k-1}$ deduced from the recurrence relations for $Q_{k}$ and $r_{k}$. Next,

$$
z_{k+1, k+1}=-\frac{1}{a_{k+1}}\left(-\left(\lambda-b_{k}\right) \frac{Q_{k} r_{k+1}}{a_{1} a_{2} \cdots a_{k}}+\frac{Q_{k-1} r_{k+1}}{a_{1} a_{2} \cdots a_{k-1}}\right)=r_{k+1} \frac{Q_{k+1}}{a_{1} a_{2} \cdots a_{k+1}}
$$

and so on for $z_{l, k+1}, l>k+1$. Thus the form of the matrix $B$ is correct. Now we decompose $B$ into two parts: $B=B_{1}+B_{2}$, where $B_{1}$ and $B_{2}$ are lower and upper triangular matrices with the same diagonal elements $\left(B_{1}\right)_{i, i}=$ $\left(B_{2}\right)_{i, i}=\frac{1}{2} B_{i, i}$. For the estimation of norms of matrices we use the formula

$$
\|K\|=\sup _{\|x\| \leq 1} \sup _{\|y\| \leq 1}|(y, K x)| .
$$

We have for two finite vectors $x=x_{0} e_{0}+x_{1} e_{1}+\cdots+x_{n} e_{n}$ and $y=y_{0} e_{0}+$ $y_{1} e_{1}+\cdots+y_{n} e_{n}$ :

$$
\begin{aligned}
\left(y, B_{1} x\right)= & \frac{1}{2} \overline{b_{0,0}} y_{0} \overline{x_{0}} h_{0}^{2}+\left(\overline{b_{1,0}} y_{1} \overline{x_{0}}+\frac{1}{2} \overline{b_{1,1}} y_{1} \overline{x_{1}}\right) h_{1}^{2} \\
& +\cdots+\left(\overline{b_{n, 0}} y_{n} \overline{x_{0}}+\overline{b_{n, 1}} y_{n} \overline{x_{1}}+\cdots+\frac{1}{2} \overline{b_{n, 1}} y_{n} \overline{x_{n}}\right) h_{n}^{2} .
\end{aligned}
$$

Here, we denote by $b_{i, j}$ the elements of the matrix $B$. Then

$$
\begin{aligned}
\left|\left(y, B_{1} x\right)\right| & \leq \frac{1}{2} \sum_{k=0}^{n}\left|b_{k, k}\left\|x_{k}\right\| y_{k}\right| h_{k}^{2}+\sum_{j=1}^{n} \sum_{k=0}^{n-j}\left|b_{k+j, k}\left\|x_{k}\right\| y_{k+j}\right| h_{k+j}^{2} \\
& =\frac{1}{2} \sum_{k=0}^{n}\left|b _ { k , k } \left\|x_{k}\left|\left\|y_{k}\left|h_{k}^{2}+\sum_{j=1}^{n} \sum_{k=0}^{n-j}\right| b_{k+j, k} \frac{h_{k+j}}{h_{k}}\right\| x_{k} h_{k} \| y_{k+j} h_{k+j}\right|\right.\right. \\
& \leq C\left(\frac{1}{2}+q+q^{2}+\cdots+q^{n}\right)\|x\|\|y\| \\
& \leq C \frac{1-q^{n+1}}{1-q}\|x\|\|y\| .
\end{aligned}
$$

A similar estimation is applicable for the matrix $B_{2}$. The first part of the theorem is thus proved.

To prove that for $\lambda \in \Omega(A)$ the estimation (10) holds, one can use the known theorem on the decay rates of the inverse of banded matrices (see [5]) and the matrix representation of $B=(\lambda I-A)^{-1}$ obtained in the first part of our proof. 


\section{Application to Padé approximants}

In this section we consider some applications of Theorem 1. First of all we note that Theorem 1 together with Propositions 1 and 2 gives a characterization of the resolvent set of $A$ in terms of the polynomials $P_{n}$ and $Q_{n}$. On the other hand this theorem also gives a result on the convergence of the continued fractions (5). We recall that the fraction $P_{n} / Q_{n}$ is a diagonal type Padé approximant for $\phi(\lambda)$ at infinity (see, e.g., Theorem 7.15 (B) in [8, page 250]).

Corollary 2. If $\lambda \in \Omega(A)$, then the remainder of Padé approximation in linear form tends to zero, i.e.,

$$
\lim _{n \rightarrow \infty}\left[Q_{n}(\lambda) \phi(\lambda)-P_{n}(\lambda)\right] h_{n}=0
$$

Proof. We have $z^{(0)}=R(\lambda) e_{0}$ and $\phi(\lambda)=\left(R(\lambda) e_{0}, e_{0}\right)=z_{0,0}$, with $h_{0}=1$. On the other hand $z_{0,0}=\gamma Q_{0}-P_{0}=\gamma$; hence $\phi(\lambda)=\gamma$ and the corollary follows from Proposition 2.

Remark 1. In the case of a symmetric operator $A$ with a representation (1) in some orthogonal basis, the polynomials

$$
q_{n}=Q_{n} / d_{n}, \quad d_{n}=\gamma_{0} \gamma_{1} \cdots \gamma_{n-1}
$$

are orthonormal and if $\lambda \in \Omega(A)$, then $q_{n}(\lambda) \phi(\lambda)-p_{n}(\lambda) \rightarrow 0$ as $n \rightarrow \infty$. In this case we have $h_{n}=1 /\left|d_{n}\right|$.

Corollary 3. If there exists a positive constant $C_{1}$ such that $\left|\alpha_{k}\right|,\left|\gamma_{k}\right| \leq C_{1}$, then for all $\lambda \in \Omega(A)$

$$
\limsup _{n \rightarrow \infty}\left|Q_{n}(\lambda) h_{n}\right|^{1 / n}>1 \text {. }
$$

Proof. Both $Q_{n}$ and $r_{n}$ satisfy the same recurrence relation (6). Hence

$$
Q_{n-1} r_{n}-Q_{n} r_{n-1}=-a_{1} a_{2} \cdots a_{n-1},
$$

or equivalently

$$
Q_{n-1} h_{n-1} \frac{r_{n}}{h_{n} a_{1} \cdots a_{n}} a_{n} \frac{h_{n}}{h_{n-1}}-Q_{n} h_{n} \frac{r_{n-1}}{h_{n-1} a_{1} \cdots a_{n-1}} \frac{h_{n-1}}{h_{n}}=-1 .
$$

From Theorem 1 we get

$$
\left|\frac{r_{k}}{h_{k} a_{1} \cdots a_{k}}\right| \leq C q^{k}, \quad q<1,
$$

so the sequence $Q_{n} h_{n}$ cannot be majorized by a geometric sequence $p^{n}$ with $p<1 / q$, i.e., for any $p<1 / q$ and any positive constant $C$ the inequality

$$
\left|Q_{n}(\lambda) h_{n}\right| \leq C p^{n}
$$

is not satisfied for an infinite number of indices $n$. For a subsequence $\Lambda \subset \mathbb{N}$ we thus have

$$
\left|Q_{n}(\lambda) h_{n}\right| \geq C p^{n}, \quad n \in \Lambda ;
$$

and consequently if we choose $p$ such that $1 / q>p>1$ we have

$$
\limsup _{n \rightarrow \infty}\left|Q_{n}(\lambda) h_{n}\right|^{1 / n} \geq p>1
$$

giving the required result. 
Remark 2 . In the symmetric case this corollary gives us the well-known characterization of $\Omega(A)$ in terms of the orthonormal polynomials $q_{n}$ : if $\lambda \in \Omega(A)$, then $\lim \sup _{n \rightarrow \infty}\left|q_{n}(\lambda)\right|^{1 / n}>1$ ([11]).

The combination of these two corollaries gives

Theorem 2. If $A$ is bounded and $0<\left|\alpha_{k}\right|,\left|\gamma_{k}\right| \leq C_{1}<\infty$ for some constant $C_{1}$, then for any $\lambda \in \Omega(A)$ there exists a subsequence of the Pade approximants $\pi_{n}=P_{n}(\lambda) / Q_{n}(\lambda)$ which converges with a geometric rate to $\phi(\lambda)$.

Proof. From Corollary 1 we have

$$
\lim _{n \rightarrow \infty} Q_{n} h_{n} \phi(\lambda)-P_{n} h_{n}=0 .
$$

Let $\Lambda \subset \mathbb{N}$ be such that

$$
\lim _{n \rightarrow \infty}\left|Q_{n}(\lambda) h_{n}\right|^{1 / n}>1
$$

then $\left|Q_{n}(\lambda) h_{n}\right| \geq C p^{n}$ for some $p>1$. Hence

$$
\left|\phi(\lambda)-\frac{P_{n}(\lambda)}{Q_{n}(\lambda)}\right| \leq C\left(\frac{q}{p}\right)^{n},
$$

which proves the theorem.

Remark 3. In the symmetric case this statement seems to be unnoticed. Indeed, suppose $A$ is in the form (1) in an orthonormal basis, and consider the so-called asymptotically periodic case (or the corresponding limit-periodic continued fraction) where for some $N$ we have

$$
\lim _{k \rightarrow \infty} \alpha_{k N+i}=\delta_{i}, \quad i=0,1,2, \ldots N-1,
$$

and similarly for $\beta_{k N+i}$ and $\gamma_{k N+i}$. Then it is known that the poles of the Padé approximants $\pi_{n}=P_{n} / Q_{n}$ are essentially concentrated on a system of $N$ closed intervals ([4], [7]). The convergence of $\pi_{n}$ however depends on the behaviour of the so-called spurious poles. Nevertheless our theorem shows that for any $\lambda$ in the exterior of the spectrum some subsequence of $\pi_{n}$ converges with a geometric rate.

Finally we note that from Theorem 1 we get a connection between the fact that $\lambda \in \Omega(A)$ and the convergence of Padé approximants $\pi_{n}(\lambda)=P_{n}(\lambda) / Q_{n}(\lambda)$. The convergence of $\pi_{n}(\lambda)$, without further requirements on the rate, does not imply that $\lambda \in \Omega(A)$. A counterexample is given by the spectral measure $d \mu(x)=\sqrt{1-x^{2}} d x$ on $[-1,1]$, for which $P_{n}(x)=2^{-n+1} U_{n-1}(x)$ and $Q_{n}(x)=2^{-n} U_{n}(x)$, where $U_{n}(x)$ are the Chebyshev polynomials of the second kind. At $\lambda=1 \in \sigma(A)$ we have $\pi_{n}(1)=2 n /(n+1)$ which converges, but without geometric rate. The convergence of some subsequence with a geometric rate is also not sufficient to imply that $\lambda \in \Omega(A)$. A counterexample is given by any symmetric measure on $[-1,1]$, because then at $\lambda=0 \in \sigma(A)$ we always have $P_{2 n}(\lambda)=0$.

The following question is thus of interest: does the convergence of the whole sequence $\pi_{n}(\lambda)$ with a geometric rate imply that $\lambda \in \Omega(A)$ ? 


\section{REFERENCES}

1. N. I. Akhiezer, The classical moment problem, Fizmatgiz, Moscow, 1961; English transl., Oliver and Boyd, Edinburgh.

2. N. I. Akhiezer and I. M. Glazman, Theory of linear operators in Hilbert space, Nauka, Moscow, 1966; English transl., Pitman, Boston.

3. A. I. Aptekarev, Asymptotic properties of polynomials orthogonal on a system of contours and periodic motions of Toda lattices, Mat. Sb. 125 (167) (1984), 231-258; English transl., Math. USSR-Sb. 53 (1986), 233-260.

4. A. I. Aptekarev and E. M. Nikishin, The scattering problem for a discrete Sturm-Liouville operator, Mat. Sb. 121 (163) (1983), 327-358; English transl., Math. USSR-Sb. 49 (1984), 325-355.

5. S. Demko, W. F. Moss, and P. W. Smith, Decay rates for the inverse of band matrices, Math. Comp. 43 (1984), 491-499.

6. J.S. Geronimo and K.M. Case, Scattering theory and polynomials orthogonal on the real line, Trans. Amer. Math. Soc. 258 (1980), 467-494.

7. J.S. Geronimo and W. Van Assche, Orthogonal polynomials with asymptotically periodic recurrence coefficients, J. Approx. Theory 46 (1986), 251-283.

8. W.B. Jones and W.J. Thron, Continued fractions: Analytic theory and applications, Encyclopedia Math. Appl., vol. 11, Addison-Wesley, Reading, MA, and Cambridge Univ. Press, Cambridge, 1980.

9. M.A. Krasnoselskii and M.G. Krein, Fundamental theorems on the extension of Hermitian operators and certain of their applications to the theory of orthogonal polynomials and the problem of moments, Uspekhi Mat. Nauk 2(19) (1947), no. 3, 60-106. (Russian)

10. E.M. Nikishin, Discrete Sturm-Liouville operators and some problems of function theory, Trudy Sem. Petrovsk. 10 (1984), 3-77; English transl., J. Soviet Math. 35 (1986), 2679-2744.

11. E.M. Nikishin and V.N. Sorokin, Rational approximations and orthogonality, Nauka, Moscow, 1988; English transl., Transl. Math. Monographs, vol. 92, Amer. Math. Soc., Providence, RI.

12. W. Van Assche, Asymptotics for orthogonal polynomials and three-term recurrences, Orthogonal Polynomials: Theory and Practice (P. Nevai, ed.), NATO-Adv. Sci. Inst. Ser. C: Math. Phys. Sci., vol. 294, Kluwer Academic Publishers, Dordrecht, 1990, pp. 435-462.

Keldysh Institute of Applied Mathematics, Russian Academy of Science, Miusskaya SQ. 4, 125047 Moscow, RussiA

E-mail address: aptekaaCapplmat.msk.su

Department of Mathematics, Nizhnil Novgorod State University, Gagarina 23A, NizHNII Novgorod, RUSSIA

E-mail address: yuas@appl.nnov.su

Department of Mathematics, Katholieke Universiteit Leuven, CelestijnenlaAn 200 B, B-3001 HeVERLEE, BELGIUM

E-mail address: walter@wis.kuleuven.ac.be 